\title{
Lapita. Ancêtres océaniens. Oceanic Ancestors de Christophe Sand et Stuart Bedford (éd.)
}

\section{Gilles Bounoure}

\section{(2) OpenEdition}

\section{Journals}

Édition électronique

URL : http://journals.openedition.org/jso/6443

DOI : $10.4000 /$ jso. 6443

ISSN : 1760-7256

Éditeur

Société des océanistes

\section{Édition imprimée}

Date de publication : 15 décembre 2011

Pagination : 424-426

ISBN : 978-2-85430-037-4

ISSN : 0300-953x

\section{Référence électronique}

Gilles Bounoure, «Lapita. Ancêtres océaniens. Oceanic Ancestors de Christophe Sand et Stuart Bedford (éd.) », Journal de la Société des Océanistes [En ligne], 133 | 2e semestre 2011, mis en ligne le 31 décembre 2011, consulté le 21 septembre 2020. URL : http://journals.openedition.org/jso/6443; DOI : https://doi.org/10.4000/jso.6443

Ce document a été généré automatiquement le 21 septembre 2020.

(c) Tous droits réservés 


\title{
Lapita. Ancêtres océaniens. Oceanic Ancestors de Christophe Sand et Stuart Bedford (éd.)
}

\author{
Gilles Bounoure
}

\section{RÉFÉRENCE}

SAND Christophe et Stuart BEDFORD (éds), 2010. Lapita. Ancêtres océaniens. Oceanic

Ancestors, Paris, Somogy-Musée du quai Branly, 304 pages, chronologie, bibliogr., cartes, très nombreuses illustrations.

1 Avec ce volume publié à l'occasion d'une remarquable exposition présentée au musée du quai Branly du 9 novembre 2010 au 9 janvier 2011, on ne saurait trop se réjouir de disposer d'une revue détaillée des principales recherches récentes concernant «la tradition Lapita ", ses vestiges archéologiques et les hypothèses qu'ils autorisent. Édité en version bilingue (texte français à gauche, anglais à droite) et rédigé par des auteurs pour la plupart renommés, il devrait toucher un public plus large que les publications spécialisées rendant compte habituellement de ce type d'investigation, dont le caractère technique restreint forcément le lectorat. Et comme près de la moitié des textes concernent des trouvailles ou des fouilles effectuées au Vanuatu et en NouvelleCalédonie (où cette tradition a pris son nom), «terrains» dont Stuart Bedford et Christophe Sand sont d'éminents spécialistes, l'ouvrage devrait aussi rencontrer un écho particulier auprès des habitants de cette extrémité orientale de l'arc mélanésien.

2 Après une introduction des éditeurs ("Lapita, icône archéologique du premier peuplement austronésien du Pacifique Sud-Ouest»), le volume offre seize chapitres, parfois suivis d'exposés complémentaires, répartis en quatre grandes sections. La première fixe le "cadre général», Christophe Sand exposant «les origines océaniennes: historique de la recherche sur la tradition Lapita " puis "l'origine du nom 'Lapita' ", Jim Allen et James O'Connell faisant part des « données récentes sur le 
premier peuplement de la Mélanésie», Matthew Spriggs s'attachant à décrire «la néolithisation de l'Asie du Sud-Est insulaire ", chapitre complété par une mise au point de Geoffrey Irwin sur «les pirogues et la navigation Lapita», Andrew Pawley présentant pour finir les «origines et différenciation des langues austronésiennes de l'Asie du Sud-Est à l'Océanie lointaine ». Cette section est accompagnée de cartes, de schémas et de photographies des trouvailles les plus spectaculaires d'Indonésie et de Mélanésie occidentale.

Illustrée de cartes, de paysages et d'objets, la deuxième section (« le développement du Lapita ») ne comprend que deux chapitres, «L'émergence de l'ensemble culturel Lapita dans l'archipel de (sic) Bismarck » par Glenn R. Summerhayes, qui lui a adjoint une synthèse sur "les sources et la répartition de l'obsidienne Lapita ", et celui de Peter Sheppard, intitulé "Dans le Grand Océan : l'arrivée des Lapita en Océanie lointaine », suivi d'un résumé concernant «la poterie Lapita des îles Reef/Santa Cruz, dans le sudest des îles Salomon» dû à Scarlett Chiu. La troisième section ("traditions Lapita d'Océanie lointaine ») est la plus développée et sans doute celle que les océanistes trouveront la plus neuve ou saisissante. Elle s'ouvre sur «Chaîne d'îles; la présence Lapita dans le nord du Vanuatu » chapitre dû à Stuart Bedford et Jean-Christophe Galipaud, que ce dernier a complété d'une note sur « Makué et Shokraon : peuplement initial et évolution des cultures dans le nord du Vanuatu». Stuart Bedford, Mattew Spriggs, Hallie Buckley, Frédérique Valentin, Ralph Regenvanu et Marcellin Abong présentent conjointement leurs fouilles particulièrement riches $d$ '« un cimetière de premier peuplement: le site de Teouma, au sud d'Éfaté, au Vanuatu ", découvert en 2004.

4 À ces exposés centrés sur le Vanuatu succèdent le chapitre que consacre Frédérique Valentin aux "sépultures et pratiques funéraires du ier millénaire avant J.-C. de Mélanésie et de Polynésie occidentale», celui d'Elisabeth Matisoo-Smith sur la "génétique du peuplement insulaire: l'expansion austronésienne et les Lapita», la synthèse de Christohe Sand sur «le Lapita du sud : le cas calédonien » complétée d'une note qu'il signe avec Jacques Bolé et André Ouetcho sur « une fosse à poteries Lapita sur le site éponyme de Foué, Nouvelle-Calédonie », et le chapitre de Geoffrey Clark dévolu aux « horizons lointains : la dispersion Lapita aux îles Fidji et en Polynésie occidentale » sur lequel se conclut cette section, illustrée de cartes, de schémas, de photos de terrain et d'objets, ainsi que de dessins.

5 Les quatre chapitres de la dernière section (« dynamiques culturelles et diversifications Lapita ») sont consacrés d'abord aux « objets en coquillage Lapita et leur technique de fabrication", par Katherine Szabó et à "la technologie lithique Lapita», par P. Sheppard. La contribution de Patrick V. Kirch, "Entre récif et jardin: l'écologie et l'économie de subsistance Lapita ", est complétée par une synthèse d'Atholl Anderson sur « l'impact de la colonisation Lapita sur la faune indigène ». Christophe Sand décrit "la fin d'une époque : enracinements et diversifications culturelles issues du Lapita » en conclusion de cette partie, illustrée de photographies de sites et d'objets qui leur sont associés. Avant l'abondante bibliographie (11 pages) qui clôt le volume, une chronologie suggère que les auteurs du volume sont tombés d'accord pour situer « vers 1300 avant J.-C. » l'arrivée des premiers groupes de langue austronésienne à l'extrémité occidentale de l'arc mélanésien, dépassant ainsi ou mettant de côté les controverses auxquelles a pu donner lieu cette datation. 
6 Tout comme le détail des contributions, dues à des spécialistes chevronnés et maîtres dans l'art de présenter leur sujet à leurs étudiants ou à un public plus large, l'économie générale de l'ouvrage appelle peu de remarques. Les spécialistes ou les lecteurs attentifs ne seront pas surpris de retrouver dans plusieurs de ces textes des développements dont ils auront déjà lu la substance ailleurs: ainsi de ceux de Christophe Sand, largement inspirés de sa synthèse sur le Lapita calédonien (2010) ${ }^{1}$ éditée par la Société des Océanistes, et qu'on devra préférer comme largement plus précise et démonstrative que les résumés qu'il livre ici. S'il y a lieu de regretter que les sites réputés les plus anciens, ceux de l'archipel Bismarck et notamment Mussau, d'où a pu partir « l'expansion Lapita » en direction de «l'Océanie lointaine », de la Polynésie occidentale et sans doute aussi de la Micronésie, ne soient pas décrits plus minutieusement, il convient de rappeler que les archéologues se sont heurtés là à maintes difficultés d'identification et de datation qui ont retardé la publication de leurs trouvailles. Certains partis pris d'écriture, comme la notation scientifique 103 pour désigner les millénaires (p. 46), n'ont peut-être pas leur place dans un volume destiné aux non spécialistes, si justifiés qu'ils soient d'un autre point de vue.

7 C'est peut-être pour sa couverture (!) que l'ouvrage mériterait un réel reproche, celui d'énoncer des promesses ou un programme qu'il ne tient pas. Elle s'orne d'un photomontage associant une poterie Lapita gravée de médaillons, une tête maori en bois pourvue de ses tatouages, un motif de natte du Vanuatu et le détail d'une "pirogue à balancier, photo Jacques Viot " (pour être plus précis que les éditeurs, une embarcation de Manokwari, à l'orée occidentale de la Cenderawasih Bay de la Nouvelle-Guinée occidentale, reconnaissable à sa figure de proue) suggère que ce volume pourrait offrir au lecteur des aperçus sur ces arts témoignant de l'influence à longue portée de la « culture Lapita » dans une large part de l'Océanie... et n'en est rien. Cette influence est admise par la plupart des spécialistes de ces arts mais aussi des archéologues, à commencer par Chr. Sand qui écrivait ailleurs : «l'héritage Lapita est également visible en termes de structures graphiques et techniques développées dans les arts océaniens " $(2010$, p. 18 , voir aussi p. 227 , etc.) et elle justifiait ce genre de « concept de couverture " que n'a su ou n'a pu illustrer ce volume. C'est à peine s'il mentionne la «tradition du tatouage » attestée par l'existence de peignes Lapita (p. 14) et la survivance de « règles graphiques de base de la tradition Lapita... dans les productions de Fidji et de la Polynésie occidentale » (p. 26, sous la plume de Chr. Sand), sans rien dire des motifs de natte, de tatouage ou de sculpture affichés en couverture. Mais s'il s'agissait du programme d'une future exposition "Lapita » s'intéressant à ces arts, il faudrait en saluer l'annonce à deux mains, comme le mérite déjà ce livre collectif qui rend accessible à beaucoup de lecteurs les conclusions les plus récentes et sûres de l'archéologie océanienne. 


\section{BIBLIOGRAPHIE}

SAND Christophe, 2010. Lapita calédonien. Archéologie d'un premier peuplement insulaire océanien, Paris, Société des Océanistes, Travaux et documents océanistes 2.

\section{NOTES}

1. Liste non exhaustive des passages de Lapita. Ancêtres océaniens (LAO) repris ou résumés du Lapita calédonien (LC) : LAo pp. 30-38 = LC pp. 21-31, LAo pp. 192-196 = LC pp. 53 sq., 93 sq., 113 sq., LAO pp. 202-204 = LC pp. 169 sq., 173 à 190, LAO pp. 204-208 = LC pp. 195-210. 